\title{
Gas Signatures from Cultured Neutrophils and Peripheral Blood Mononuclear Cells Obtained from Healthy Humans
}

Hye-Won Shin*,\#, Brandon J. Umber*, Simone Meinardi, Szu-Yun Leu, Frank Zaldivar, Donald R. Blake\# and Dan M. Cooper\#

Department of Pediatrics University of California, Irvine, Irvine, CA 92697

\#Contributed Equally

\begin{abstract}
We hypothesized that leucocytes may be a significant source of physiologically active gases. Furthermore, it is hoped that alterations by disease of these volatile organic compound (VOC) profiles can ultimately be used as noninvasive biomarkers. We recently demonstrated that transformed promyelocitic cells produce distinct gases. (J Transl Med. 2009 7:31). The current study extends this work to neutrophils and peripheral blood mononuclear cells (PBMCs). Cells were isolated from the peripheral blood of healthy donors ( $n=10,18-65 \mathrm{yrs}$ old), resuspended in RPMI, and incubated in bioreactors for $24 \mathrm{hrs}$. The headspace was analyzed using gas chromatography. Acetaldehyde was elevated from neutrophils(median (min, max); $197(34,577)$ ppbv) compared with media $(88$ $(40,116)$ ppbv, $p=0.014)$. In the presence of alanine, neutrophils emitted more acetaldehyde $(>1.5$ fold compared to basal level). In contrast, acetaldehyde from PBMCs was $26(8,89)$ ppbv, significantly below media $(p=0.004)$. Adding alanine did not affect acetaldehyde emissions from PBMCs. Also, hexanaldehyde appeared metabolized by neutrophils and PBMCs. This study demonstrates 1) that human primary immune cells produce measurable VOCs in vitro, and 2) the ability to detect basal levels of acetaldehyde from unperturbed cultured neutrophils. Moreover, the data suggest that different leukocyte subtypes have different VOC profiles.
\end{abstract}

Keywords: Gas biomarkers; VOCs; Leukocytes; Bioreactor; Neutrophils; Peripheral blood mononuclear cells; Headspace gas

\section{Introduction}

There is growing recognition that many of the volatile organic compounds detectable in exhaled breath reflect disease or metabolism $[1,2]$ However, attempts to identify disease-specific breath biomarkers for clinical application face numerous challenges. One of the most salient of these challenges is linking gas signals detected in the breath to cellular mechanisms. Given, for example, the associations now established between the detection of exhaled gases like nitric oxide and increased lung eosinophilia [3,4] we reasoned that a better understanding of the gas profiles produced by other circulating inflammatory cells would prove useful in efforts to gauge the clinical utility of gases detected in exhaled breath.

Compared to the widespread focus on gases like nitric oxide and asthma cited above, there has been far fewer investigations attempting to link the production of distinct gases to specific cell types [5-8]. For example, Deng et al. [9], using solid-phase microextraction combined with gas chromatography-mass spectrometry (GC-MS), demonstrated that whole blood collected from patients with lung cancer generated appreciable amounts of hexanaldehyde and heptanaldehyde compared to whole blood from healthy donors. Moreover, in our own laboratory, we recently demonstrated that distinct gases such as acetaldehyde and hexanaldehdye were produced specifically by transformed promyelocitic cells [10].

In this study, we hypothesized that human leukocytes found in the circulating blood (neutrophils and PBMCs) could generate detectable levels of cell type-specific gasses. We utilized a bioreactor developed for our previous study [10], and a gas chromatographic system capable of detecting gas concentrations down to parts-per-trillion. The goals of the current study were two fold: 1) to examine the adaptability of the bioreactors and the analytical techniques using human leukocyte cells in culture; and 2) to identify unique gas signatures that could distinguish neutrophils from PBMCs.

\section{Methods}

Subjects: Ten healthy, adult men between the ages of 18 and $65 \mathrm{yr}$ participated in this study through the Institute for Clinical Translational Sciences (ICTS) general blood donor program. Subjects having a history of any chronic medical conditions, or use of any medications were excluded from the study. The Institutional Review Board at the University of California, Irvine approved the study.

Blood sampling and cell isolation: The blood was sampled from the antecubital vein using EDTA-treated vacutainers. Neutrophils and PBMCs were then isolated using OptiPrep Density Gradient Medium (Sigma). The isolation of the neutrophils and PBMCs consistently yielded $\geq 98 \%$ purification based on Wright-giemsa stain and Flow Cytometric analysis [11,12].

Cell culture and headspace gas collection: Purified neutrophils and PBMCs were resuspended in RPMI 1640 [Irvine Scientific, Santa Ana, CA] and supplemented with $10 \%$ fetal calf serum (FCS). The vials containing cell suspensions $\left(30 \times 10^{6}\right.$ cells $/ 10 \mathrm{~mL}$ ) were placed inside bioreactors specifically designed to collect the gaseous headspace above aqueous cultures [6] (see Figure 1). To minimize ambient contaminants each bioreactor was flushed with whole air containing low levels of VOCs and $5 \% \mathrm{CO}_{2}$ for 10 minutes at $125 \mathrm{~mL}$ per minute.

${ }^{*}$ Corresponding author: Hye-Won Shin, PhD, Department of Pediatrics, 1303A Hewitt Hall, University of California, Irvine, CA 92697, Tel:949-824-3702; Fax: 949824-336; E-mail: hyewons@uci.edu

Received June 08 Accepted August 22, 2011; Published August 26, 2011

Citation: Shin H, Umber BJ, Meinardi S, Leu S, Zaldivar F, et al. (2011) Gas Signatures from Cultured Neutrophils and Peripheral Blood Mononuclear Cells Obtained from Healthy Humans. J Mol Biomark Diagn 2:112. doi:10.4172/21559929.1000112

Copyright: (c) 2011 Shin $\mathrm{H}$, et al. This is an open-access article distributed under the terms of the Creative Commons Attribution License, which permits unrestricted use, distribution, and reproduction in any medium, provided the original author and source are credited 


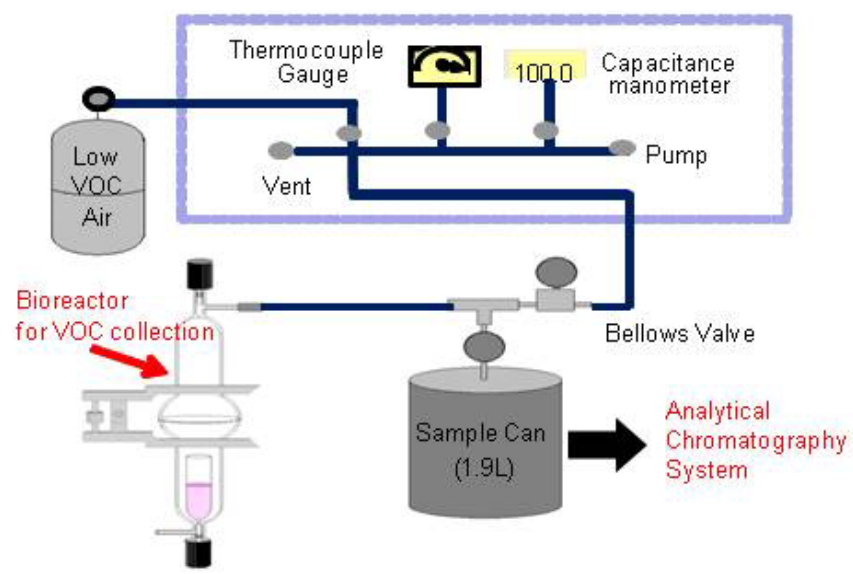

Figure 1: The $378 \mathrm{~mL}$ glass bioreactor designed for incubating cells in air containing low levels of volatile organic compounds and post incubation collection of the gaseous headspace. The analysis of headspace gases and room samples was performed on the system developed by Blake-Rowland Laboratory to quantify trace gases, and is described in detail elsewhere [13].

The bioreactors were then placed in an incubator at $37^{\circ} \mathrm{C}$ for $24 \mathrm{hrs}$. The headspace gases were collected after incubation and analyzed using a gas chromatographic system with multiple column/detector combinations [13]. Following sample collection the bioreactor was disassembled and the cells were immediately collected and counted. Trypan blue exclusion assay was performed to determine the percentage of live cells following incubation.

Gas chromatography: The analyses of the headspace gases were performed on the system previously developed by the Blake-Rowland Laboratory at UCI to measure trace atmospheric gases. A complete description of the GC parameters and analytical methods are given elsewhere [10,13] .Briefly, a $233 \mathrm{~cm}^{3}$ (at STP) sample was cryogenically pre-concentrated and injected into a multi-column/detector GC system. The system consists of three Hewlett-Packard 6890 GC units (Wilmington, DE) using a combination of columns and detectors capable of separating and quantifying hundreds of gases, including, but not limited to, non-methane hydrocarbons (NMHCs), alkyl nitrates and halocarbons in the ppbv to pptv range $\left(10^{-9}-10^{-12}\right)$. The detectors include flame ionization detectors (FIDs), electron capture detectors (ECDs), and a mass spectrometer detector (MSD). Preliminary identifications of the unknown compounds were made using GC-MSD and verification was obtained by injecting the diluted headspace of pure compounds (Sigma-Aldrich, St. Louis, MO) to ensure that both the elution time and the mass spectrum matched that of the unknown. The percent $\mathrm{CO}_{2}$ in the air used to flush the bioreactors was determined using a Carbosphere 80/100 packed column output to a thermal conductivity detector (TCD).

\section{Statistical analysis}

From each of the 10 samples of neutrophils, PBMCs, and corresponding media, the gases were obtained with or without replicates depending on the availability of blood or media, and the average was calculated for each gas if there were replicates. The gases whose maximum expression from all samples was less than $100 \mathrm{pptv}$ and/or whose maximum change, increase or decrease, from media was less than $50 \%$ were considered not scientifically meaningful and were excluded for further analysis. The remaining gases from neutrophils and from PBMCs were compared to the corresponding media using the Wilcoxon signed-rank test. Due to the large number of gases under investigation, we further applied Benjamini and Hochberg's False Discovery Rate (FDR) controlling method to adjust for multiple comparisons. A gas from neutrophils or PBMCs is considered significantly changed from the media if $\mathrm{FDR}<0.05$. All analysis was done with SAS 9.2 (Cary, NC) and data are presented with median and range (minimum, maximum) due to the small sample size and the nonparametric test applied to the data. The mean and standard deviation (s.d.) is also provided in bracket.

\section{Results}

A total of 59 gases were obtained from human neutrophils $(n=10$, total replicates $=20)$ and PBMCs $(n=10$, total replicates $=28)$ and media control $(n=10$, total replicates $=22)$, of which 24 gases were included for statistical analysis. After multiple comparison adjustment, 4 gases (acetaldehyde, hexanaldehyde, 3-methylpentane, methylcyclopentane) from both neutrophils and PBMCs, 3 gases (n-hexane, styrene, 2-methy2-butene) from only PBMCs, and 1 gas (ethylene sulfide) from only neutrophils were found significantly different from media. To exclude gases originating from other than cells, the experiments included
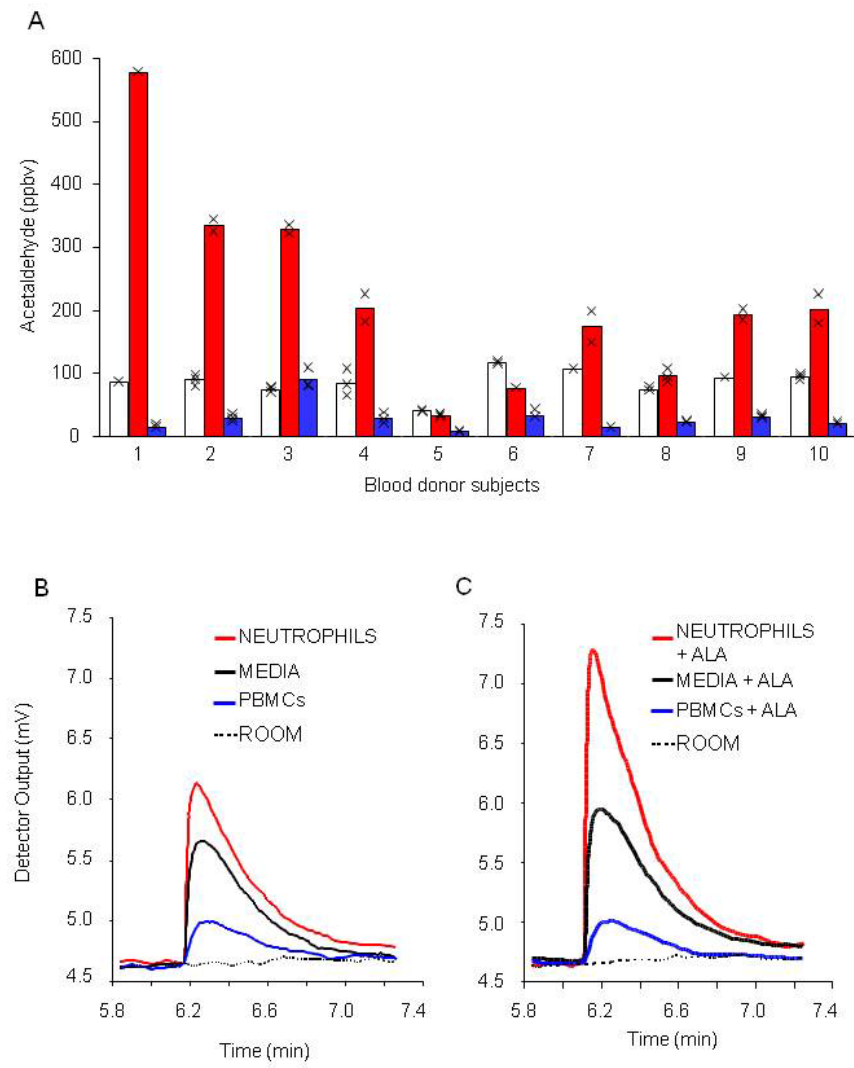

Figure 2: (A) The acetaldehyde emitted in the bioreactor headspace of neutrophils (red bar) and PBMCs (blue bar) following $24 \mathrm{~h}$ of incubation. Acetaldehyde concentration is significantly higher from neutrophils $(p=0.014)$ and significantly lower from PBMCs compared to media (white bar, $p=0.004$ ). X represents replicates. (B) Representative chromatograms of acetaldeyde from neutrophils (in red), PBMCs (in blue), and culture media (black). Acetaldehyde has been generated from neutrophils, however, PBMCs from the same subject presented a level below the culture medium. (C) Representative chromatograms of acetaldeyde in the presence of an additional $100 \mathrm{mg} / \mathrm{L}$ alanine. Neutrophils cultured with additional alanine (in red) emitted 1.5 fold higher amounts of acetaldehyde when compared to the neutrophils from the same subject cultured in basal medium. PBMCs from the same subject presented a level below the culture medium even in the presence of alanine (in blue). 


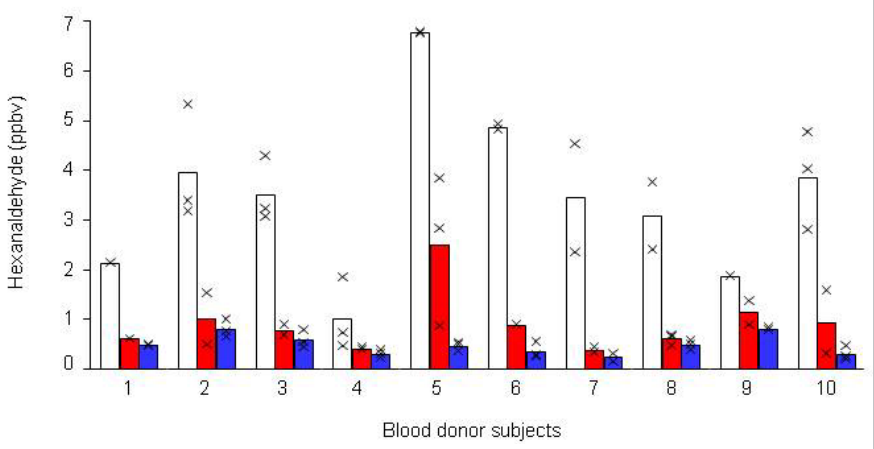

Figure 3: Hexanaldehyde emitted in the bioreactor headspace of neutrophils (red bar) and PBMCs (blue bar) following $24 \mathrm{~h}$ of incubation are presented $X$ represents replicates. The hexanaldehyde concentrations are significantly lower from neutrophils and PBMCs compared to media (white bar).

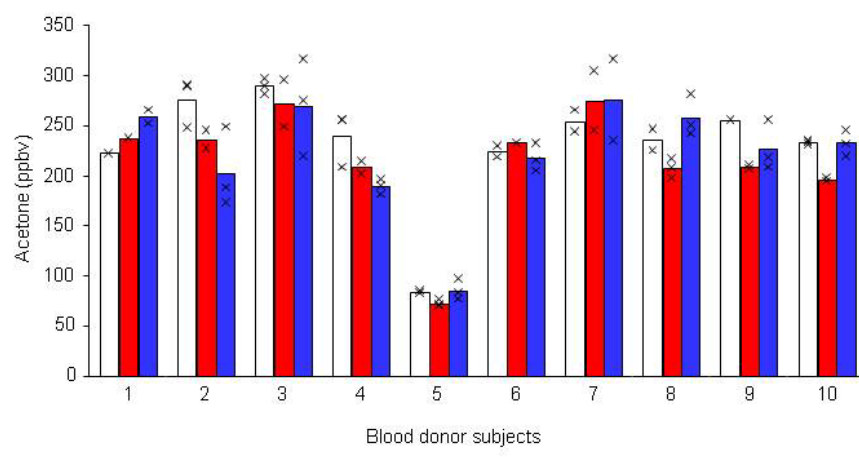

Figure 4: The acetone concentration emitted in the bioreactor headspace of neutrophils (red bar) and PBMCs (blue bar) following $24 \mathrm{~h}$ of incubation are presented. $X$ represents replicates. The emitted acetone concentrations from cell culture bioreactors in which the neutrophils and PBMCs were grown were not different from the headspace gases obtained in the media only (white bar).

the analyses of various control conditions such as ambient room air, incubator air, headspace gas from empty flushed and incubated bioreactors, headspace gas from empty EDTA-treated vacutainers, and headspace gas from incubated density gradient solutions used for leukocyte separation.

Neutrophils and PBMCs produced several distinct gas signatures that were significantly different from media control. Figure $2 \mathrm{~A}$ illustrates a significantly increased emission of acetaldehyde from cultured neutrophils isolated from ten healthy blood donors (median (min, max): $197(34,577)$ ppbv; [mean \pm s.d: $221 \pm 159$ ppbv]) when compared with media alone $(88(40,116)[86 \pm 21] \mathrm{ppbv}, \mathrm{p}=0.014, \mathrm{FDR}=0.038)$. Interestingly, the level of acetaldehyde from PBMCs obtained from the same subjects was $26(8,89)[29 \pm 23]$ ppbv, significantly below media alone $(\mathrm{p}=0.004, \mathrm{FDR}=0.015)$. An overlay of representative chromatograms of acetaldehyde from neutrophils, PBMCs, and culture media are presented in Figure 2B. The concentration of acetaldehyde in the headspace of the neutrophil culture was greater than the culture medium. However, the headspace of PBMCs cultured from the same subject had concentrations of acetaldehyde below the culture medium. As described elsewhere the tailing peak is due to the oxygenate's interaction with the surfaces and column of the analytical system[10]. Neutrophils emitted 1.5 fold higher amounts of acetaldehyde in the presence of an additional $100 \mathrm{mg} / \mathrm{L}$ alanine. In contrast, the addition of alanine did not make any changes in acetaldehyde released from cultured PBMCs (Figure 2C).
Hexanaldehyde was observed to decrease in the bioreactor headspace with both neutrophils and PBMCs. Hexanaldehyde was 3.5 $(1.0,6.8)[3.4 \pm 1.6]$ ppbv from media, but was only $0.8(0.4,2.5)[0.9$ $\pm 0.6] \mathrm{ppbv}$ from neutrophils $(\mathrm{p}=0.002, \mathrm{FDR}=0.011)$ and $0.5(0.2,0.8)$ $[0.5 \pm 0.2] \mathrm{ppbv}$ from PBMCs $(\mathrm{p}=0.002$. $\mathrm{FDR}=0.011)$. We speculate that the decrease in concentration of this gas may be due to removal by cellular metabolism. Hexanaldehyde concentrations from neutrophils and PBMCs in culture obtained from the 10 donors are presented in Figure 3.

Ethylene sulfide was $1.4(0.3,2.6)[1.3 \pm 0.7] \mathrm{ppbv}$ from media, the level was significantly lower $(\mathrm{p}=0.0098, \mathrm{FDR}=0.033)$ from neutrophils $0.6(0.1,2.0)[0.9 \pm 0.7]$ ppbv but not from PBMCs $1.8(0.1,4.6)[1.8 \pm$ 1.6] ppbv (data not shown).

Acetone was observed in the headspace of neutrophils and PBMCs in culture. However, the emitted acetone concentrations from neutrophils and PBMCs were not different from the headspace gases obtained in the media only (Figure 4).

Several gases were initially observed to be significantly higher from PBMCs and/or neutrophils in culture. However, these gases turned out to be carry-over contaminants originating from the plastic EDTA vacutainers used to collect the whole blood. These gases include methylcyclopentane, 3-methylpentane, and n-hexane as shown in Figure 5, and 2-methy-2-butene and styrene not shown.

The cell viability or percent survival for the neutrophils and PBMCs following $24 \mathrm{hr}$ incubation was $76 \pm 18 \%$ and $95 \pm 18 \%$ respectively. Neutrophils, as expected, were not as robust as the PBMCs and their population was reduced following $24 \mathrm{hr}$ incubation.

\section{Discussion}

This study demonstrates 1) that human neutrophils and PBMCs produce volatile gases that can be measured in vitro in trace amounts, 2) for the first time the ability to detect basal levels of acetaldehyde produced by unperturbed cultured neutrophils, and 3) of most significance the data suggests that different leukocyte subtypes have different gas emission profiles.

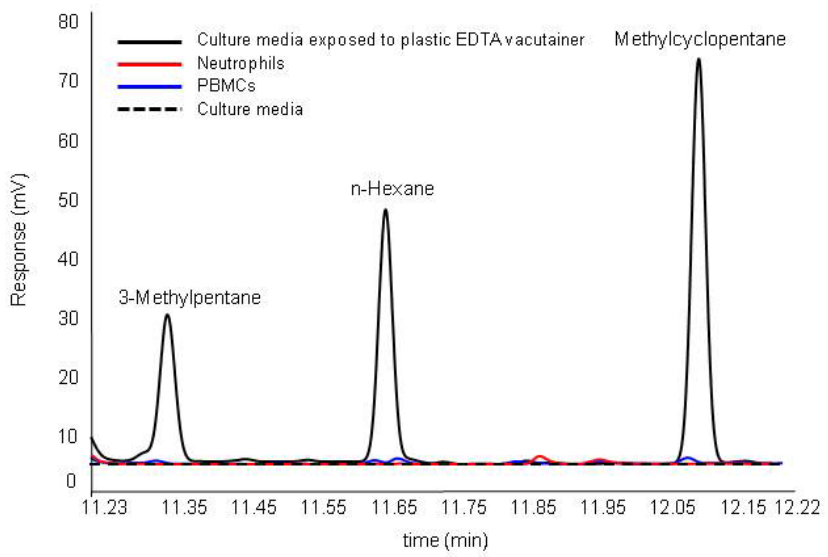

Figure 5: Representative chromatograms of media (dashed) and media exposed to plastic EDTA vacutainers. These gases, 3-methyl pentane, n-hexane, and methylcyclopentane were observed to be significantly higher from PBMCs (in blue line) and/or neutrophils (in red line) in culture. However they were determined to be carry-over contaminants from the plastic EDTA vacutainers, and excluded from the study. 
Acetaldehyde has previously been detected in exhaled human breath [14,15] and in human lung cancer cell line cultures[6-8]. In general, acetaldehyde concentrations were found to be taken up by lung cancer cell lines and normal lung cell lines. We previously demonstrated that malignant myelocytic white blood cells, using cultured HL60 cells, are capable of producing acetaldehyde [10]. The current study also demonstrates that primary myelocytic cells, i.e., neutrophils obtained from healthy subjects, emitted appreciable amounts of acetaldehyde into the gas phase, above the background media acetaldehyde levels (Figure 2). Although 2 subjects have lower acetaldehyde from neutrophils than from media (see Figure 2A), the difference (acetaldehyde from neutrophils minus acetaldehyde from media) is relatively small and the difference among subjects is expected since we obtained blood cells from normal healthy donors. Elegant work by Hazen and colleagues confirmed the ability of PMAactivated neutrophils to oxidize amino acids and produce aldehydes, a reaction requiring myeloperoxidase (MPO), hydrogen peroxide $\left(\mathrm{H}_{2} \mathrm{O}_{2}\right)$, and chloride ion $\left(\mathrm{Cl}^{-}\right)[16,17]$. Since human neutrophils have high myeloperoxidase protein expression and activity, this amino acid oxidation is a likely pathway for the generation of acetaldehyde from neutrophils. In agreement with Hazen, when the amino acid alanine was added to the culture system, acetaldehyde release from neutrophils in culture increased. We speculate that the subjects having lower levels of acetaldehyde in the headspace above neutrophils in culture (subject \#5 and \#6) may have individual differences in their metabolisms that lead to lower levels of produced acetaldehyde (i.e., lower levels of the available substrate alanine to form acetaldehyde).

In contrast, PBMCs from healthy individuals seemed to consume acetaldehyde rather than release it into the gas phase. The acetaldehyde released from cultured cells appears to be cell type-specific (i.e, neutrophil- type vs PBMC-type). Furthermore, the addition of alanine did not alter acetaldehyde emission from PBMCs in culture. The precise mechanism of acetaldehyde metabolism by PBMCs has not yet been fully investigated.

Cultured promyelocytic HL60 cells generated appreciable amount of hexanaldehyde into the head space [10]. In addition, elevated hexanaldehyde has been detected in whole blood from lung cancer patients compared to the healthy controls [18] Intriguingly, the current study suggests that both primary neutrophils and PBMCs appear to metabolize hexanaldehyde (Figure 3). The cellular mechanisms of hexanaldehyde metabolism by human primary neutrophils and/or PBMCs require further investigation.

Our study demonstrated that ethylene sulfide was consumed in the presence of neutrophils in culture. Ethylene sulfide has not been reported in the exhaled human breath. Our observation may suggest that human neutrophils may be able to metabolize ethylene sulfide.

Several hydrocarbons of interest in exhaled breath research, such as acetone, ethane, and n-pentane were not generated nor consumed by the neutrophils and PBMCs. They were seen in the samples containing neutrophils, PBMCs, and media. However, the concentrations from cell culture flasks in which the neutrophils and PBMCs were grown were not significantly different from the headspace obtained from the media only. Figure 4 illustrates this point with acetone (ethane and n-pentane data not shown). These hydrocarbons have been detected in the exhaled human breath [19-25], and have been proposed as a means of assessing in vivo lipid peroxidation. Specifically, higher levels of exhaled acetone were observed in association with diabetes [22]. Additionally, increases in ethane concentration was observed from rat hepatocytes in the presence of oxidants [26]. The results of the current study suggest that healthy human immune cells either are not the cellular sources responsible for these gases or are under a minimal level of oxidative stress and are therefore not producing these gases under the conditions of these experiments.

Several observed VOCs, initially presenting promise as immune cell type-specific gases, turned out to be carry-over contaminants, i.e. methylcyclopentane, 3-methylpentane, n-hexane, and 2-methyl2-butene (see Figure 5, 2-methy-2-butene not shown). Although the observed methylcyclopentane was most likely associated with plastic EDTA vacutainers, it is interesting that methylcyclopentane has been detected in headspace of lung cancer cell line (NCI-H2087), $\sim 0.1 \mathrm{ppb} / 1$ $\mathrm{X} 10^{6}$ cells (8). Styrene was previously identified as a contaminant emanating from the plastic culture ware, and was excluded [10]

\section{Conclusion}

The current study demonstrates that different types of human leukocytes produce specific VOCs. The analysis of VOCs from leukocyte cells in culture may enhance our understanding of exhaled breath gases by providing the cellular source and mechanism of VOCs release. These data also provide the beginning of an inventory of VOCs associated with leukocytes from healthy people-the necessary first step from which an exploration of aberrant cells can follow. In particular, further investigations into the gas signatures of the PBMC subtypes (e.g.,monocytes and lymphocytes) will be required as will a better understanding of how typical immune cell responses (e.g., activation) alters the gas signature of these dynamic cells.

\section{Acknowledgements}

This work was supported by grants from the Pacific Southwest Regional Center of Excellence for Biodefense \& Emerging Infectious Diseases Research (U54Al065359) and from the National Center for Research Resources (NCRR), a component of the National Institutes of Health $(\mathrm{NIH})$ and the NIH Roadmap for Medical Research (UL1 RR031985). We thank the Institute for Clinical Translational Research Center (ICTS) at University of California, Irvine.

\section{References}

1. Galassetti Pr, Novak B, Nemet D, Rose-Gottron C , Cooper D M , et al. (2005) Breath Ethanol And Acetone As Indicators Of Serum Glucose Levels: An Initial Report. Diabetes Technol Ther 7: 115-123.

2. Kamboures MA, Blake DR, Cooper DM, Newcomb RL, BarkerM, et al. (2005) Breath Sulfides and Pulmonary Function In Cystic Fibrosis. Proc Natl Acad Sc USA 102: 15762-15767.

3. Jatakanon A, Lim S, Kharitonov SA, Chung KF, BarnesPJ (1998) Correlation Between Exhaled Nitric Oxide, Sputum Eosinophils, and Methacholine Responsiveness In Patients With Mild Asthma. Thorax 53: 91-95.

4. Payne DN, Adcock IM, Wilson NM, OatesT, Scallan M, et al. (2001) Relationship Between Exhaled Nitric Oxide And Mucosal Eosinophilic Inflammation In Children With Difficult Asthma, After Treatment With Oral Prednisolone. Am J Respir Crit Care Med 164: 1376-1381.

5. Chen X, Xu F, Wang Y, PanY, Lu D, et al. (2007) A Study Of The Volatile Organic Compounds Exhaled By Lung Cancer Cells In Vitro For Breath Diagnosis. Cancer 110: 835-844.

6. Filipiak W, Sponing A, Filipiak A, Ager C, Schubert J, et al. (2010) Td-Gc-Ms Analysis Of Volatile Metabolites Of Human Lung Cancer And Normal Cells In Vitro. Cancer Epidemiol Biomarkers Prev 19: 182-195.

7. Filipiak W, Sponring A, Mikoviny T, Ager C, SchubertJ, et al. (2008) Release Of Volatile Organic Compounds (Vocs) From The Lung Cancer Cell Line CALU-1 in Vitro. Cancer Cell Int 8: 17.

8. Sponring A, Filipiak W, Mikoviny T, Ager C, Schubert J, et al. (2009) Release of Volatile Organic Compounds From The Lung Cancer Cell Line NCl-H2087 In Vitro. Anticancer Res 29: 419-426.

9. Deng C, Zhang X, Li N (2004) Investigation of Volatile Biomarkers In Lung Cancer Blood Using Solid-Phase Microextraction and Capillary Gas 
Citation: Shin H, Umber BJ, Meinardi S, Leu S, Zaldivar F, et al. (2011) Gas Signatures from Cultured Neutrophils and Peripheral Blood Mononuclear Cells Obtained from Healthy Humans. J Mol Biomark Diagn 2:112. doi:10.4172/2155-9929.1000112

Chromatography-Mass Spectrometry. J Chromatogr B Analyt Technol Biomed Life Sci 808: 269-277.

10. Shin H W, Umber B J, Meinardi S, Leu SY, Zaldivar F, et.al. (2009) Acetaldehyde And Hexanaldehyde From Cultured White Cells.J Transl Med 7: 31.

11. Radom-Aizik S, Zaldivar F Jr, Leu SY, Cooper DM (2009)A Brief Bout Of Exercise Alters Gene Expression And Distinct Gene Pathways In Peripheral Blood Mononuclear Cells Of Early- and Late-Pubertal Females. J Appl Physio 107: 168-175.

12. Radom-Aizik S, Zaldivar F Jr, Oliver S, Galassetti P, Cooper DM (2010) Evidence For MicroRNA Involvement in exercise-Associated Neutrophil Gene Expression Changes. J Appl Physiol 109: 252-261.

13. Colman JJ, Swanson AL, Meinardi S, Sive BC, Blake DR,et.al (2001) Description Of The Analysis Of A Wide Range Of Volatile Organic Compounds In Whole Air Samples Collected During PEM-Tropics A and B. Anal Chem 73: 3723-3731.

14. Turner C, Spanel P, SmithD (2006) A Longitudinal Study Of Ethanol And Acetaldehyde in The exhaled breath Of Healthy Volunteers Using SelectedIon Flow-Tube Mass Spectrometry. Rapid Commun Mass Spectrom 20: 61-68.

15. Fuchs P, LoesekenC, Schubert JK, MiekischW(2010) Breath Gas Aldehydes as Biomarkers of Lung Cancer. Int J Cancer 126: 2663-2670.

16. Hazen SL, Hsu FF, D'avignon A, Heinecke JW (1998) Human Neutrophils Employ myeloperoxidase to Convert alpha-amino Acids to a battery of reactive aldehydes: a Pathway for aldehyde generation at sites of inflammation. Biochemistry 37: 6864-6873.

17. Hazen SL, D'avignon A, Anderson MM, Hsu FF, Heinecke JW (1998) Human neutrophils employ the myeloperoxidase-hydrogen Peroxide-Chloride System to oxidize alpha-amino Acids to a family of reactive aldehydes. Mechanistic studies identifying labile Intermediates along the reaction pathway. J Biol Chem 273: 4997-5005

18. DengC, Li N, ZhangX (2004) Development of headspace solid-phase microextraction with on-fiber derivatization for determination of hexanal and heptanal in human Blood. J Chromatogr B Analyt Technol Biomed Life Sci 813 47-52

19. Paredi P, Kharitonov SA, Leak D, Shah PL, Cramer D, et al. (2000) Exhaled Ethane Is Elevated In Cystic Fibrosis And Correlates With Carbon Monoxide Levels And Airway Obstruction. Am J RespirCrit Care Med 161: 1247-1251.

20. Paredi P, Kharitonov SA, Leak D, Ward S, Cramer D, et al. (2000) Exhaled Ethane, A Marker Of Lipid Peroxidation, Is Elevated In Chronic Obstructive Pulmonary Disease. Am J Respir Crit Care Med162: 369-373.

21. Paredi P, Kharitonov SA, BarnesPJ( 2000) Elevation Of Exhaled Ethane Concentration in Asthma. Am J Respir Crit Care Med 162: 1450-1454.

22. LeeJ, Ngo J, Blake,D, Meinardi S, Pontello AM, et.al(2009) Improved Predictive Models For Plasma Glucose Estimation From Multi-Linear Regression Analysis Of Exhaled Volatile Organic Compounds. J Appl Physiol 107: 155-160.

23. Kneepkens CM, Ferreira C, Lepage G, Roy CC(1992) The Hydrocarbon breath test in the Study Of Lipid Peroxidation: Principles And Practice. Clinlnvest Med 15: 163-186.

24. Kanoh S, Kobayashi H, Motoyoshi K (2005) Exhaled Ethane: an In Vivo Biomarker Of Lipid Peroxidation In Interstitial lung Diseases. Chest 128: $2387-$ 2392.

25. Habib MP, Clements NC,Garewal HS(1995) Cigarette smoking and ethane exhalation in Humans. Am J Respir Crit Care Med 151: 1368-1372.

26. Smith M T, Thor H, Hartizell P, Orrenius S (1982) The Measurement Of Lipid Peroxidation In Isolated Hepatocytes. Biochem Pharmacol 31: 19-26. 